\title{
Research on the performance of multi-base buoy arrays under the condition of on-called anti-submarine and warship-helicopter cooperation
}

\author{
Yuhang Zhang ${ }^{1, \mathrm{a}}$, Jianbo $\mathrm{Ju}^{2}$ and Siqing $\mathrm{Wang}^{3}$ \\ 123 Naval Aviation University, Yantai, China
}

\begin{abstract}
In order to improve the performance of traditional on-called Anti-submarine, the method of combining the towed sonar and helicopter deployment with traditional buoy arrays for multi-base searching is proposed. Combining the acoustics principle and the actual movement characteristics of warships and helicopters, model of naval movement and multi-base passive circular buoy arrays, square buoy arrays, and triangle buoy arrays deployed by a helicopter were established. The Monte Carlo method was used to simulate and analyse the effect of the initial distance from the call point, the initial position of the submarine, the speed of the submarine economy, the size parameters of the float array and the number of deployments on the multi-base search performance under each formation. The simulation results show that the search performance of the three multi-base arrays decreases with the increase of the initial distance, the initial position of the submarine, and the speed of the submarine economy; the circular multi-base array has the best search performance, and the appropriate deployment radius and the number of passive buoys to be deployed can be selected according to different search requirements and actual operational conditions. This method is suitable for on-called anti-submarine within a certain distance, and has certain military significance for anti-submarine warfare.
\end{abstract}

\section{Introduction}

With the development of submarine in terms of noise reduction, speed increase, and anti-submarine exploration $^{[1]}$, it is increasingly difficult to meet the needs of anti-submarine warfare by relying solely on single-base sonar. Therefore, it is important to seek multi-base collaborative search methods in various forms. At present, the synergy between surface ships and antisubmarine helicopters is mostly parallel inspection or patrol search. With the continuous updating of the submersible equipment, on the basis of solving the problem of consistent frequency of synergy, the towed sonar of the surface ship can be combined with the passive buoy array to form a multi-base search submerged array. Based on the traditional buoy array method, this paper combines the factors such as acoustic attenuation and helicopter coordinated turning, and innovatively combines the moving surface ship with the anti-submarine helicopter to set up the intercepting buoy array. The multi-base sonar buoy array model of arc, straight line and polyline is established, and the simulation results of three multi-base array search potentials under different conditions are simulated and compared.

\section{Multi-base passive buoy array model for ship motion and helicopter deployment}

\subsection{Surface warship movement, helicopter movement and buoy array placement model}

The coordinate system is established with the initial position point $P_{0}$ of the surface ship and the antisubmarine helicopter as the coordinate origin. Assume that the initial approximate position of the submarine target $S$ is $P_{s u b 0}$, the coordinate is $v_{s u b 0}$, the initial speed of the submarine is $v_{s u b 0}$, the angle between the rough heading of the submarine and the horizontal coordinate axis is $\alpha$, and the angle between the $P_{0} P_{\text {sub } 0}$ and the horizontal coordinate axis is $\beta_{1}$.

The time when the ship acquires the information of the submarine to be dispatched is $t_{\text {sta }}$, the time taken for the helicopter to take off is $t_{t-o}$, the time required to fly to the first buoy point of the search area is $t_{f l i}$, and the time required to deploy the buoy in the search area Is $t_{\text {buoy }}$. The anti-submarine helicopter starts from $P_{0}$, and

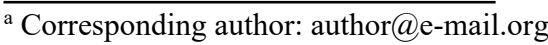


the average flight speed after synthesizing with the wind speed is $v_{h e l}$, and the gravity acceleration of the mission water is $g$.

Let the distance between $P_{0}$ and $P_{1}$ be $L_{o}$, the total number of passive buoys placed is $n$, the position of each buoy point is $P_{i}$, the flight distance from $P_{i} \rightarrow P_{i+1}$ is $L_{i}$, and the corresponding direct flight time is $t_{i}$.When the intercepting array is arranged, the distances of adjacent buoy points are usually equal, and the coordinated turning time of the helicopter at each buoy point is $t_{\text {turn }}$. Then the total time from the acquisition of submarine information to the end of the anti-submarine helicopter deployment buoy is:

$$
t_{\text {total }}=t_{\text {sta }}+t_{t-o}+L_{o} / v_{\text {hel }}+(n-1)\left(L_{i} / v_{\text {hel }}+t_{\text {turn }}\right)
$$

The surface ship departs from $P_{0}$ at a speed of $v_{n a v}$ and heads in direction $P_{0} \rightarrow P_{s u b 0}$. According to the principle of time synergy, the anti-submarine helicopter deploys the sonar buoy array and the towed sonar decentralization simultaneously. When the towing sonar is lowered, the surface ship arrives at $O_{s}\left(x_{s h i 0}, y_{s h i 0}\right)$. At this time, the towed sonar began to work in an active manner, and combined with the buoy array to multi-base search for the potential, sailing at speed $v_{\text {sea }}$ along $O_{s} P_{s u b 0}$ until $P_{s u b 0}$.

\subsection{Buoy array and size requirements for arcs, straight and broken lines}

When the anti-submarine helicopter receives the order, it starts from $P_{0}$ and arrives at $P_{1}$ to place the first buoy, and then lays the buoy $P_{i}$ in turn. Fig. 2, Fig. 3 and Fig. $4^{[2]}$ are ship route diagrams of the arc buoy array, the linear buoy array and the three-fold line buoy array respectively.

When the buoy array is deployed, the rough heading of the submarine is generally used as the central axis of the formation, so that the angular division of the detection angle $\omega$ coincides with the central axis, and the buoys are arranged at equal intervals according to the array in the detection angle range. The key to deployment is to determine the size parameter $R$ of the interception array and the placement of the first buoy. The size parameter $R$ must be satisfied that the submarine cannot escape the buoy array even if it is evaded by its maximum maneuverer speed $V_{\text {submax }}$ before the buoy array is released. Then:

$$
V_{\text {submax }} \cdot t_{\text {total }} \leq R
$$

Through the geometric relationship of each formation, the relationship between $L_{o}, L_{i}$ and size parameter $R$ can be obtained, and the value of $R$ needs to satisfy these relations. The placement of the first buoy is determined by $L_{o}$ and the initial heading angle $\beta_{2}$ of the helicopter ${ }^{[3]}$.
$R$ can be obtained according to the geometric relationship, the triangle sine theorem, the triangle cosine theorem and the induction formula ${ }^{[4]}$. For arc multi-base buoy array, the corresponding radius of the arc is the size parameter $R$, then:

$$
L_{i}=2 R \sin \frac{\varphi}{2}=2 R \sin \frac{\omega}{2(n-1)}
$$

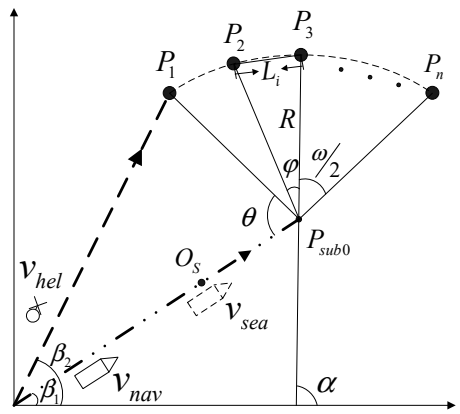

Figure 2. Warship and helicopter movement model under round multi-base buoy array.

$$
\begin{aligned}
& L_{0}=\sqrt{S_{0}^{2}+R^{2}+2 S_{0} R \cos \left(\alpha-\beta_{1}+\omega / 2\right)} \\
& \beta_{2}=\beta_{1}+\operatorname{arc} \frac{R \sin \left(\alpha-\beta_{1}+\omega / 2\right)}{\sqrt{S_{0}^{2}+R^{2}+2 S_{0} R \cos \left(\alpha-\beta_{1}+\omega / 2\right)}}
\end{aligned}
$$

For straight line multi-base buoy array, $P_{s u b 0}$ to the linear array distance is the size parameter $R$, then:

$$
L_{i}=\frac{2 R \tan (\omega / 2)}{n-1}
$$

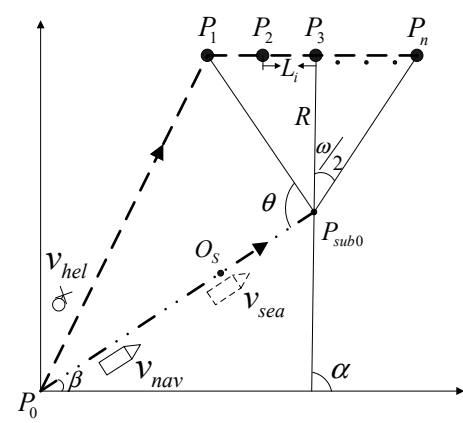

Figure 3. Relationship between size parameters and float spacing of square multi-base buoy array.

$$
\begin{gathered}
L_{0}=\sqrt{S_{0}^{2}+\left(\frac{R}{\cos \omega / 2}\right)^{2}+2 S_{0} R \frac{\cos \left(\alpha-\beta_{1}+\omega / 2\right)}{\cos \omega / 2}} \\
\beta_{2}=\beta_{1}+\operatorname{arc} \frac{R \sin \left(\alpha-\beta_{1}+\omega / 2\right)}{\sqrt{S_{0}^{2} \cos ^{2}(\omega / 2)+R^{2}+S_{0} R U}}
\end{gathered}
$$

Among them, $U=\cos \left(\alpha-\beta_{1}+\omega\right)+\cos \left(\alpha-\beta_{1}\right)$.

For polyline multi-base buoy array, the distance from $P_{\text {sub } 0}$ to the edge of any line is the size parameter $R$, and the opening and closing angle of the line array is $\gamma$, then: 


$$
\begin{gathered}
L_{0}=\sqrt{S_{0}^{2}+\left(\frac{R}{\sin \frac{\gamma+\omega}{2}}\right)^{2}+2 S_{0} R \frac{\cos \left(\alpha-\beta_{1}+\frac{\omega}{2}\right)}{\sin \frac{\gamma+\omega}{2}}} \\
\beta_{2}=\beta_{1}+\operatorname{arc} \frac{R \sin \left(\alpha-\beta_{1}+\frac{\omega}{2}\right)}{\sqrt{S_{0}^{2} \sin ^{2} \frac{\gamma+\omega}{2}+R^{2}+2 S_{0} R V}}
\end{gathered}
$$

of the towed sonar under the influence of the blind zone is $D_{t}$, and the passive buoy detection distance is $D_{b}$ when there is no active sound source.

Based on the bistatic sonar equation: $T L_{1}+T L_{1}=S L$ $N L+D I-D T+T S$ under the condition of noise limitation, if the formula (17),(18),(19) is satisfied ${ }^{[6]}$, then:

$$
\begin{aligned}
& \left\{\begin{array}{l}
\left|P_{i} S_{k}\right|=\sqrt{\left(x_{\text {buoi }}-x_{\text {subk }}\right)^{2}+\left(y_{\text {buoi }}-y_{\text {subk }}\right)^{2}} \\
\left|O_{q} S_{k}\right|=\sqrt{\left(x_{\text {shiq }}-x_{\text {subk }}\right)^{2}+\left(y_{\text {shiq }}-y_{\text {subk }}\right)^{2}} \\
Q=S L-N L+D I-D T+T S_{k}-120 \\
20 \lg \left|P_{i} S_{k}\right|+20 \lg \left|O_{q} S_{k}\right|+\alpha\left(\left|P_{i} S_{k}\right|+\left|O_{q} S_{k}\right|\right) \leq Q
\end{array}\right. \\
& \left|P_{i} S_{k}\right| \leq D_{t} \text { or }\left|P_{i} S_{k}\right| \leq D_{b}
\end{aligned}
$$

Among them, $V=\cos \left(\alpha-\beta_{1}+\omega / 2\right) \sin \frac{\gamma+\omega}{2}$.

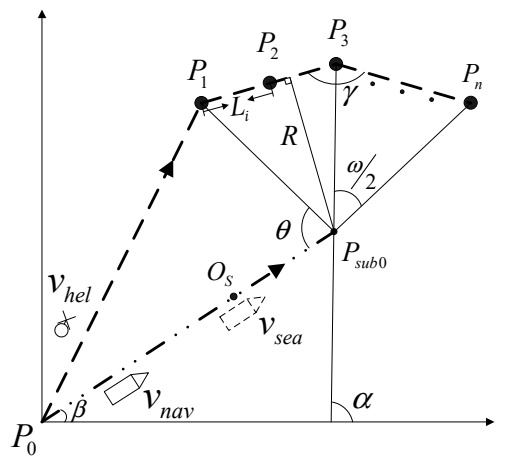

It is considered that the multi-base sonar buoy array searches for the submarine. According to the Monte Carlo method if the total number of times the submarine is searched is $M$, the probability of searching for the multi-base sonar buoy array $P$ is:

$$
P=\frac{M}{Q K} \times 100 \%
$$

\subsection{Simulation and analysis}

In order to visually and accurately reflect the solution gap, the ocean area covered by the three multi-base sonar buoy arrays is equal when comparing the potentials of the schemes ${ }^{[7]}$. Set $t_{s t a}+t_{t-o}=0.25 \mathrm{~h}$, the average speed of the anti-submarine helicopter to the calling area, the buoy and the monitoring search is $150 \mathrm{~km} / \mathrm{h}$, the surface speed of the surface ship is 20 knots, the search speed is 12 knots, and the gravity acceleration in the sea area is $9.8 \mathrm{~m} / \mathrm{s}^{2}$, the multi-base cooperative frequency is $1.5 \mathrm{kHz}$, corresponding to the seawater absorption coefficient $\alpha=0.0946$, the sound source level $S L=220 \mathrm{~dB}$, the detection valve is $D T=14 d B$, the noise level $N L=70 \mathrm{~dB}$, and the directivity index is $D I=0 \mathrm{~dB}$. For each coordinated turning time $1.5 \mathrm{~min}$, the buoy is passively omnidirectional, the effective working distance is $3 \mathrm{~km}, \beta=45^{\circ}, \omega=90^{\circ}, \gamma=120^{\circ}$, and the submarine's heading distribution is $8^{\circ}$, and $R$ is taken as $35 \mathrm{~km}$.In order to simplify the analysis, the probability density of the target strength of the submarine is as follows ${ }^{[8]}$ :

$$
f(x)=\left\{\begin{array}{l}
-4 \times 10^{-3}(x-15)^{2}+0.1(10 \leq x \leq 15) \\
-10^{-3}(x-15)^{2}+0.1(15 \leq x \leq 25)
\end{array}\right.
$$

The multi-base search potential pattern of the three buoy arrays is simulated and the potential of the search potential is simulated to analyse the influence of different conditions on the search potential.

\subsubsection{Parameter setting and simulation results}

According to Table 1, the parameters are set. Each group will study the influence of the interval parameters as variables on the search potential. The simulation results are shown in Fig. 5. 
Table 1. Parameter settings.

\begin{tabular}{|c|c|c|c|c|c|c|}
\hline & $\begin{array}{c}\text { Initial } \\
\text { Distance }(\mathrm{km})\end{array}$ & $\begin{array}{c}\text { Initial Position } \\
\text { Distribution }(\mathrm{km})\end{array}$ & $\begin{array}{c}\text { Initial Speed } \\
(\mathrm{kn})\end{array}$ & $\begin{array}{c}\text { Initial Heading } \\
\text { Angle }\end{array}$ & $\mathrm{R}(\mathrm{km})$ & Number of buoys \\
\hline Sim1 & $40-90$ & 3 & 5 & $45^{\circ}$ & 35 & 8 \\
\hline Sim2 & 50 & $1-5$ & 5 & $45^{\circ}$ & 35 & 8 \\
\hline Sim3 & 50 & 3 & $4-12$ & $45^{\circ}$ & 35 & 8 \\
\hline Sim4 & 50 & 3 & 5 & $0^{\circ}-180^{\circ}$ & 35 & 8 \\
\hline Sim5 & 60 & 3 & 7 & $45^{\circ}$ & $30-40$ & 8 \\
\hline Sim6 & 50 & 3 & 7 & $45^{\circ}$ & 35 & $3-8$ \\
\hline
\end{tabular}

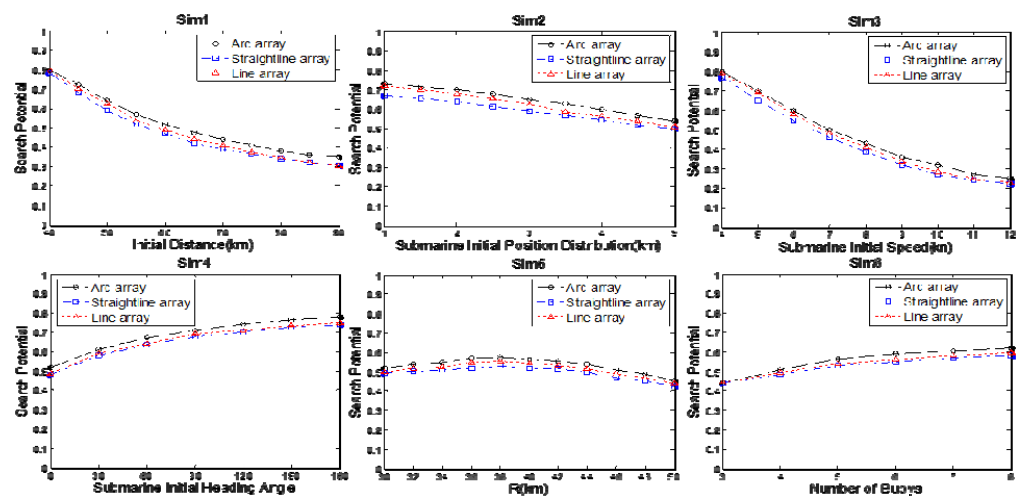

Figure 5. Simulation results.

\subsubsection{Analysis and conclusion}

It can be seen from Fig. 5 that under the same conditions, the search performance of the arc multi-base array is better than that of the straight line array and the polyline array. And the search efficiency of the three multi-base sonar buoy arrays decreases with the initial distance, the initial position distribution of the submarine, and the initial speed of the submarine, and increases with the initial heading angle of the submarine. At the same time, it can be seen in the figure that when the number of passive buoys is fixed, the probability of searching for each formation will increase and then decrease with the increase of the size parameter. under the condition that the size parameter is certain, the search potential probability first increases with the number of layouts, and gradually becomes gentle. Therefore, for different search requirements, combined with actual combat conditions, it is necessary to select the appropriate deployment radius and number of placements, so as to improve the search potential while ensuring better economy.

\section{Conclusion}

This paper proposes a method of dragging sonar and helicopter deployment of traditional buoy array combined multi-base search potential to improve the potential of search potential. Based on the actual movement characteristics of surface ships and helicopters, this paper establishes a multi-base passive buoy array model for ship motion and helicopter deployment, and establishes a multi-base joint search potential model under arc array, linear array and polygonal line array respectively. The simulation analyzes the influence of six factors on the multi-base search potential performance under each formation. The method is applicable to the call search potential within a certain distance range, and has certain military significance for anti-submarine warfare.

\section{References}

1. Ketter $\mathrm{T}$ N. Anti-submarine warfare in the 21st century[R]. Pennsylvania: Naval Operation Centre, 2004.

2. Teruhisa Nakal. Search models with continuous effort under various criteria[J]. JORSJ, 1988,31(3):335-351.

3. Jacques Locat,Homa Lee. Submarine Mass Movements and Their Consequences: An Overview[M].Springer Berlin Heidelberg:2009-0615.

4. Garnaud Xavier,Mei Chiang C.. Bragg scattering and wave-power extraction by an array of small buoys[J]. Proceedings of the Royal Society A,2010,466(2113).

5. GARNAUD, XAVIER,MEI, CHIANG C. Wavepower extraction by a compact array of buoys[J]. Journal of Fluid Mechanics,2009,635.

6. John M. Danskin. A Helicopter Versus Submarine Search Game[J]. Operations Research,1968,16(3).

7. Wilby, Andy. Deployment of a Deep Tow Synthetic Aperture Sonar System[J]. EN,2012,53(2).

8. Forrest R N. Some notes on search, detection and localization modeling[R]. Pennsylvania: Naval Operation Centre, 1996. 